\title{
Retrieving the susceptibility from time-resolved terahertz experiments
}

\author{
J. M. Schins \\ Opto-Electronic Materials Section, DelftChem Tech, Delft University of Technology, Julianalaan 136, \\ 2628 BL Delft, The Netherlands \\ E. Hendry \\ School of Physics, University of Exeter, Stocker Road, Exeter EX4 4QL, United Kingdom \\ M. Bonn and H. G. Muller \\ FOM-Institute for Atomic and Molecular Physics, Kruislaan 407, 1098 SJ Amsterdam, The Netherlands
}

(Received 28 November 2006; accepted 26 June 2007; published online 7 September 2007)

\begin{abstract}
We present an analytical expression for the observed signal in time- and phase-resolved pump-probe studies, with particular emphasis on terahertz time-domain spectroscopy. Maxwell's equations are solved for the response of damped, harmonic oscillators to a driving probe field in the perturbative regime. Our analytical expressions agree with the one previously reported in the literature [Nemec et al., J. Chem. Phys. 122, 104503 (2005)] in the Drude limit; however, they differ in the case of a vibrational resonance. (C) 2007 American Institute of Physics. [DOI: 10.1063/1.2761915]
\end{abstract}

\section{INTRODUCTION}

The use of ultrafast lasers to unravel the dynamics of physical and chemical processes in a variety of systems, ranging from gas-phase dipoles and liquids to monolayers on substrates, is becoming increasingly widespread. The essence of such studies is that the system is brought out of its ground state by an ultrashort excitation pulse ("pump" pulse) and that the relaxation to the (new) equilibrium is followed real time by a second ("probe") pulse, which can be variably delayed with respect to the first.

In most of these experiments, the temporally integrated intensity of the probe pulse is recorded with a square law detector. As such, the phase information contained in the time-dependent probe field is lost. However, the phase of the field contains information about transient changes in the complex conductivity that is complementary to that contained in the amplitude of the field envelope. In phaseresolved experiments, one detects both the amplitude and phase shift of all components contained in the probe field either through a gating technique (e.g., time-resolved terahertz spectroscopy ${ }^{2-12}$ ) or through heterodyning the signal (e.g., two-dimensional infrared ${ }^{13}$ or interferometric photoelectron spectroscopy ${ }^{14}$ ). Though we concentrate here on the experimental analysis of time-resolved terahertz spectroscopy (TRTS) - Refs. 2-10 and 15-18 provide a partial overview of this field - the equations we derive can in principle be applied to any phase-resolved spectroscopy. In addition to the explicit consideration of the phase, the theoretical treatment of these experiments also has to take into account the varying sample response over the range of frequency components contained within the probe pulses.

There have been a number of notable previous efforts in this area, ranging from numerical (e.g., using the finitedifference time-domain pulse propagation method to simulate time-resolved terahertz experiments ${ }^{19}$ ) to analytic approaches with varying degrees of complexity. ${ }^{20-22}$ More recently, a quite general analytical theory was developed by Nemec et al. showing how to extract the transient (third order) conductivity from the experimental data without ad hoc assumptions; ${ }^{23}$ subsequent publications from the same group showed how to interpret the measured data in the framework of the most frequently used models. ${ }^{1,24}$

In this contribution we further generalize the above mentioned work, ${ }^{1,23,24}$ specifically to accommodate lifetimes of the excited state that are small compared with its scattering time.

\section{EXPERIMENTAL SETUP}

The central features of a TRTS experimental setup are shown schematically in Fig. 1. A single laser beam is divided into three parts: (i) a pump beam, used to photoexcite the sample; (ii) a generation beam, to generate a terahertz pulse in either a nonlinear crystal ${ }^{25}$ or a biased emitter, ${ }^{26}$ and (iii) a

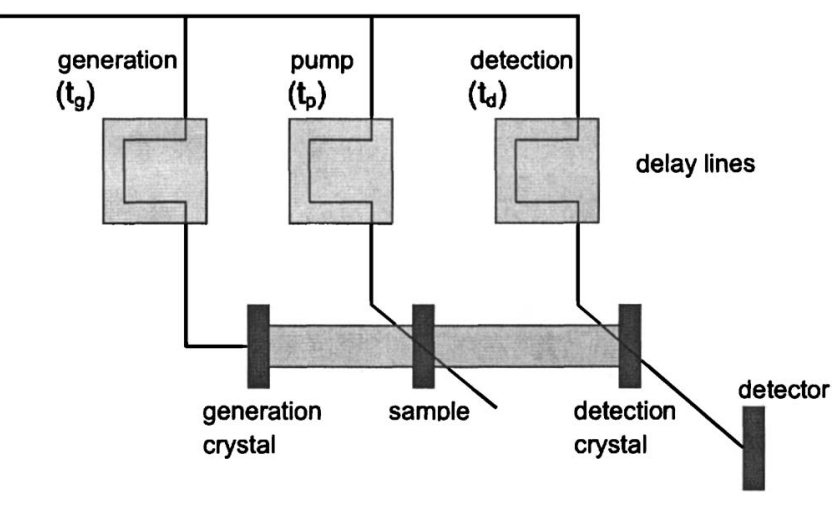

FIG. 1. Schematic of a time-resolved terahertz setup, with three delay lines to independently set the generation, pump, and detection delay times, respectively. The laser beam passing through the generation delay generates terahertz radiation. The terahertz pulse propagates to and through the sample, which is excited by a second laser beam passing through the pump delay. The transmitted terahertz pulse is sampled by means of the third laser beam passing through the detection delay. Throughout the paper we use the identities $t \equiv t_{d}-t_{g}$ and $t_{e} \equiv t_{p}-t_{g}$. 
detection beam, to detect the time-dependent wave form of the transmitted terahertz pulse by electro-optical sampling ${ }^{25}$ or using a semiconductor antenna. ${ }^{26}$ These three beams can be delayed separately, though only two of the three delays are independent as far as the experimental results are concerned.

The pump-induced differential terahertz field $\Delta E\left(z, t, t_{e}\right)$ at spatial coordinate $z$, and times $t \equiv t_{d}-t_{g}$ and $t_{e} \equiv t_{p}-t_{g}$ as defined in Fig. 1, is determined by the temporal response of the material after photoexcitation. ${ }^{20,27}$ However, when material properties change on a time scale that is comparable to the terahertz pulse duration, and different sample properties are probed by the front and tail of the terahertz pulse, the analysis is nontrivial. One of the simplest such cases to consider in transient terahertz experiments is that of a semiconductor photoexcited with a femtosecond laser pulse. This leads to a gas of mobile electrons in the conduction band of the semiconductor, the density of which first increases due to photoexcitation and then decreases due to trapping and recombination. When the time scales of the generation and decay of these mobile electrons are comparable to or faster than the duration of the terahertz pulse, it is very challenging to extract the transient photoconductivity response from the experimentally determined terahertz spectrum. ${ }^{28}$

\section{APPROXIMATIONS AND ASSUMPTIONS}

The main approximation we make in our analysis is in assuming a small, perturbative response of the system to the pump pulse; this corresponds to neglecting the interaction between the pump-induced dipoles. In the absence of the exciting pump pulse, the terahertz wave form $E_{0}(z, t)$ induces a polarization $P_{0}(z, t)$ in the sample. Due to the pump a small but significant fraction of ground-state electrons are excited, and consequently the polarization induced by the terahertz wave form changes, thus giving rise to a change in the propagated wave form $\Delta E\left(z, t, t_{e}\right)$. The specific response of photogenerated charge carriers to the terahertz field is contained within $\Delta E\left(z, t, t_{e}\right)$, which therefore contains information about the charge dynamics. The perturbative approximation applies as long as $\Delta E\left(z, t, t_{e}\right) \ll E(z, t)_{0}$ throughout the sample, which requires the radiation of the pump-induced dipoles to be sufficiently small, and the sample sufficiently thin for the accumulated phase lag between the electric fields with and without pump to remain close to zero. Then the disturbances $\Delta E$ and $\Delta P$ are linearly proportional both to the probe field and to the excitation intensity.

Other assumptions made in the analysis are (i) that the medium is isotropic, such that the electric and magnetic fields have a linear and mutually orthogonal polarization throughout the beam path; (ii) that the sample is spatially homogeneous, with a material response depending on frequency only; (iii) that the sample thickness exceeds both the dimension of the terahertz wave packet and that of the penetration depth of the pump beam (that is to say, we assume that $100 \%$ of the pump light is absorbed in the sample, and that multiple reflections within the sample do not interfere temporally with the main transmitted wave packet); (iv) that there is no dispersion of the pump beam in the sample; (v) that the excited states can be described by a collection of damped harmonic oscillators with identical force constants (i.e., we do not consider inhomogeneous line broadening); and (vi) that the excited charge density decays monoexponentially, as in a first order recombination process. These approximations still allow for an analytical treatment, without loosing applicability to a wide range of experimental studies.

\section{MAXWELL'S EQUATIONS}

Consider Maxwell's equations for electromagnetic propagation along a single spatial coordinate $z$, with the electric field $E\left(z, t, t_{e}\right)$ and polarization $P\left(z, t, t_{e}\right)$ polarized along the $x$ axis, and the magnetic field $H\left(z, t, t_{e}\right)$ along the $y$ axis. All involved fields depend parametrically on the delay times $t_{p}, t_{g}$, and $t_{d}$, for the pump, generation, and detection delays, respectively (see Fig. 1), with the definitions $t \equiv t_{d}-t_{g}$ and $t_{e} \equiv t_{p}-t_{g}$. In SI units Maxwell's equations read

$$
\begin{aligned}
& \varepsilon_{0} \frac{\partial E\left(z, t, t_{e}\right)}{\partial t}+\frac{\partial P\left(z, t, t_{e}\right)}{\partial t}+\frac{\partial H\left(z, t, t_{e}\right)}{\partial z}=0, \\
& \mu_{0} \frac{\partial H\left(z, t, t_{e}\right)}{\partial t}+\frac{\partial E\left(z, t, t_{e}\right)}{\partial z}=0 .
\end{aligned}
$$

The detector response equation relates the electric field in the sample to the measured electric field $E^{\text {meas }}\left(z, t, t_{e}\right)$ by means of the detector response function $\psi(t),{ }^{20}$

$$
\begin{aligned}
E^{\text {meas }}\left(z, t, t_{e}\right)= & \theta\left(t+T_{\text {win }}\right) \theta\left(T_{\text {win }}-t\right) \\
& \times \int_{0}^{\infty} d t^{\prime} \psi\left(t^{\prime}\right) E\left(z, t-t^{\prime}, t_{e}\right),
\end{aligned}
$$

with $\theta(\tau)$ as the Heaviside's unit step function vanishing for $\tau<0$. The two step functions determine the time window within which the terahertz field is sampled. Both the time window and the detector response function limit the resolution of the terahertz measurements.

The polarization in the sample follows directly from the average positions of the charges $x_{i}\left(t, t_{e}\right)$ and their effective charges $q_{i}$,

$$
P\left(z, t, t_{e}\right)=V^{-1} \sum_{i=1}^{N_{t}} q_{i} x_{i}\left(t, t_{e}\right),
$$

with $V$ the volume of the sample and $N_{t}$ the total number of dipoles. A small fraction of these dipoles are excited by the pump light, their number being $N_{e}$. The remaining dipoles $\left(N_{t}-N_{e}\right)$ are not affected by the pump light. Quite generally, the polarization can be written as a Green's function integral over past times as follows:

$$
P\left(z, t, t_{e}\right)=V^{-1} \int d t^{\prime} E\left(z, t^{\prime}, t_{e}\right) \sum_{i=1}^{N_{t}} \frac{q_{i}^{2}}{m_{i}} G_{i}\left(t-t^{\prime}, t_{e}\right),
$$

with $G_{i}\left(t-t^{\prime}, t_{e}\right)$ as the response of the $i$ th dipole to an instantaneous impulse $\delta\left(t-t^{\prime}\right), q_{i}$ its effective charge, and $m_{i}$ its mass. For the ground-state dipoles the temporal evolution is determined by a single Green's function $G_{g}\left(t-t^{\prime}\right)$, i.e., this function does not have a particle index $i$. The exact expres- 
sions for these Green's functions follow from Eqs. (8) through (10) below. Excited-state dipoles each have their own response, depending on how long they persist in their excited state, before relaxing back to the ground state. Using the differential field $\Delta E\left(z, t, t_{e}\right) \equiv E\left(z, t, t_{e}\right)-E_{0}(z, t)$, the polarization can now be separated into three terms,

$$
\begin{aligned}
P\left(z, t, t_{e}\right)= & V^{-1} \int d t^{\prime} E\left(z, t^{\prime}, t_{e}\right) \\
& \times\left\{\sum_{i=1}^{N_{i}-N_{e}} \frac{q_{g}^{2}}{m_{g}} G_{g}\left(t-t^{\prime}\right)+\sum_{i=1}^{N_{e}} \frac{q_{i}^{2}}{m_{i}} G_{i}\left(t-t^{\prime}, t_{e}\right)\right\} \\
= & P_{0}(z, t)+\rho_{t} \int d t^{\prime} \Delta E\left(z, t^{\prime}, t_{e}\right) \frac{q_{g}^{2}}{m_{g}} G_{g}\left(t-t^{\prime}\right) \\
& +\Delta P\left(z, t, t_{e}\right),
\end{aligned}
$$

with the total particle density $\rho_{t} \equiv N_{t} / V$, and

$$
\begin{aligned}
\Delta P\left(z, t, t_{e}\right)= & \int d t^{\prime} E\left(z, t^{\prime}, t_{e}\right) \\
& \times\left\{V^{-1} \sum_{i=1}^{N_{e}} \frac{q_{i}^{2}}{m_{i}} G_{i}\left(t-t^{\prime}, t_{e}\right)-\rho_{e} \frac{q_{g}^{2}}{m_{g}} G_{g}\left(t-t^{\prime}\right)\right\},
\end{aligned}
$$

$P_{0}(z, t)=\rho_{t} \int d t^{\prime} E_{0}\left(z, t^{\prime}\right) \frac{q_{g}^{2}}{m_{g}} G_{g}\left(t-t^{\prime}\right)$,

with $\rho_{e} \equiv N_{e} / V$. The perturbative limit mentioned in the previous section implies that the full electric field in Eq. (6) is replaced by the primary field (the electric field in the absence of sample excitation by a pump pulse),

$$
\begin{aligned}
\Delta P\left(z, t, t_{e}\right) \approx & \int d t^{\prime} E_{0}\left(z, t^{\prime}\right) \\
& \times\left\{V^{-1} \sum_{i=1}^{N_{e}} \frac{q_{i}^{2}}{m_{i}} G_{i}\left(t-t^{\prime}, t_{e}\right)-\rho_{e} \frac{q_{g}^{2}}{m_{g}} G_{g}\left(t-t^{\prime}\right)\right\} .
\end{aligned}
$$

\section{TEMPORAL PART: VIBRATIONAL RESONANCES}

The polarization is determined by the local electric field at present and past times. While focusing on the temporal part of our problem, we disregard the spatial dependence of all fields, and will include the spatial dependence in the next section when it becomes relevant again. Moreover, we replace the sum over a finite collection of dipoles with an integral over a continuous distribution.

Excited dipoles have number density $\rho_{e}$, unexcited dipoles have number density $\rho_{t}-\rho_{e}$. The dynamics of the unaffected dipoles is governed by a second order differential equation,

$$
\begin{aligned}
& L_{g}(t) x(t)=\frac{q_{g}}{m_{g}} E_{0}(t), \\
& L_{g}(t) G_{g}\left(t-t^{\prime}\right)=\delta\left(t-t^{\prime}\right),
\end{aligned}
$$

with $q_{g}$ the effective charge and $m_{g}$ the oscillating mass of a dipole with position $x(t)$, and $G_{g}\left(t-t^{\prime}\right)$ Green's function. The operator $L_{g}$ is defined as

$$
L_{g}(t)=\frac{\partial^{2}}{\partial t^{2}}+\gamma_{g} \frac{\partial}{\partial t}+\omega_{\mathrm{res} ; g}^{2},
$$

with $\gamma_{g}$ the damping rate and $\omega_{\text {res }, g}$ the resonance frequency of the ground-state dipoles.

Likewise, the excited-state dynamics are described by

$$
L_{e}(t) x(t)=\frac{q_{e}}{m_{e}} E_{0}(t)
$$

$$
L_{e}(t) G_{e}\left(t-t^{\prime}\right)=\delta\left(t-t^{\prime}\right),
$$

$$
L_{e}(t)=\frac{\partial^{2}}{\partial t^{2}}+\gamma_{e} \frac{\partial}{\partial t}+\omega_{\mathrm{res} ; e}^{2}
$$

A dipole unaffected by the pump pulse obeys Eqs. (8) and (9) at all times,

$$
x_{g}(t)=\frac{q_{g}}{m_{g}} \int d t^{\prime} E_{0}\left(t^{\prime}\right) G_{g}\left(t-t^{\prime}\right) .
$$

For excited dipoles the solution is more involved. Defining $\tau$ as the time span during which the dipole is excited, and $t_{e}$ the moment of excitation, the excited dipole obeys ground-state dynamics [Eqs. (8) and (9)] before its excitation (i.e., for times $t<t_{e}$ ), excited-state dynamics [Eq. (10)] during its excitation (i.e., for times $t_{e}<t<t_{e}+\tau$ ), and again ground-state dynamics after its decay to the ground state (i.e., for times $\left.t>t_{e}+\tau\right)$. Hence, the solution can be written as a sum of three terms, corresponding to the above mentioned time windows,

$$
\begin{aligned}
x_{e}\left(t, t_{e}, \tau\right) & \\
= & \theta\left(t_{e}-t\right) \frac{q_{g}}{m_{g}} \int_{-\infty}^{t} d t^{\prime} E_{0}\left(t^{\prime}\right) G_{g}\left(t-t^{\prime}\right) \\
& +\theta\left(t_{e}+\tau-t\right) \theta\left(t-t_{e}\right) \\
& \times\left\{\frac{q_{e}}{m_{e}} \int_{-\infty}^{t} d t^{\prime} E_{0}\left(t^{\prime}\right) G_{e}\left(t-t^{\prime}\right)-e\left(t, t_{e}\right)\right\} \\
& +\theta\left(t-t_{e}-\tau\right)\left\{\frac{q_{g}}{m_{g}} \int_{-\infty}^{t} d t^{\prime} E_{0}\left(t^{\prime}\right) G_{g}\left(t-t^{\prime}\right)+g\left(t, t_{e}, \tau\right)\right\},
\end{aligned}
$$

where $e\left(t, t_{e}\right)$ and $g\left(t, t_{e}, \tau\right)$ are solutions of the homogeneous equations of motion (see Appendix), with amplitudes determined by the condition that the particle motion is continuous both at the time of excitation $t_{e}$ and that of decay back into the ground state, $t_{e}+\tau$. Analogously, we define the timedependent effective charge, 


$$
\begin{aligned}
q_{e}\left(t, t_{e}, \tau\right)= & \theta\left(t_{e}-t\right) q_{g}+\theta\left(t_{e}+\tau-t\right) \theta\left(t-t_{e}\right) q_{e} \\
& +\theta\left(t-t_{e}-\tau\right) q_{g} .
\end{aligned}
$$

This allows for different coupling strengths of the vibrational modes with the driving field, depending on the state (excited or ground) of the oscillator. The average behavior of all excited dipoles is determined by the temporal profile of $\rho_{e}(t)$, the density of excited states. In the following we assume it has an exponential decay rate $\Gamma=1 / \tau_{c}$ :

$$
\rho_{e}(t) \equiv \rho_{e} e^{-\Gamma t} \theta(t)
$$

The polarization follows as a weighted average of the individual dipoles, each with their own lifetime $\tau$,

$$
\Delta P\left(t, t_{e}\right) \equiv \Gamma \int_{0}^{\infty} d \tau \rho_{e}(\tau)\left\{q_{e}\left(t, t_{e}, \tau\right) x_{e}\left(t, t_{e}, \tau\right)-q_{g} x_{g}(t)\right\}
$$

It is shown in the appendix that the differential polarization can be written as a sum of three terms,

$$
\Delta P\left(t, t_{e}\right)=\Delta P_{\text {driven }}\left(t, t_{e}\right)+\Delta P_{\text {excitation }}\left(t, t_{e}\right)+\Delta P_{\text {decay }}\left(t, t_{e}\right) .
$$

These terms have a straightforward interpretation:

(i) the term $\Delta P_{\text {driven }}\left(t, t_{e}\right)$ corresponds to the driven motion of the oscillators in their excited state, during the time interval $t_{e}<t<t_{e}+\tau$,

(ii) the term $\Delta P_{\text {excitation }}\left(t, t_{e}\right)$ corresponds to the damped transients (free induction decay) created at the time of excitation $t=t_{e}$; and

(iii) the term $\Delta P_{\text {decay }}\left(t, t_{e}\right)$ corresponds to the damped transients created at the (exponentially distributed) times of decay $t=t_{e}+\tau$.

Using the Fourier convention,

$$
\begin{aligned}
& f(\omega)=\int d t e^{i \omega t} f(t), \\
& f(t)=\frac{1}{2 \pi} \int d \omega e^{-i \omega t} f(\omega),
\end{aligned}
$$

the first two terms can be Fourier transformed to

$$
\begin{aligned}
& \Delta P_{\text {driven }}\left(\omega, \omega_{e}\right)+\Delta P_{\text {excitation }}\left(\omega, \omega_{e}\right) \\
& =E_{0}\left(\omega+\omega_{e}\right) \frac{\rho_{e}}{\Gamma+i \omega_{e}}\left\{\frac{q_{g}}{m}\left(q_{e}-q_{g}\right) G_{g}\left(\omega+\omega_{e}\right)+\frac{q_{e}}{m}\right. \\
& \left.\quad \times\left[q_{e}-q_{g} \frac{G_{g}\left(\omega+\omega_{e}\right)}{G_{e}\left(\omega+\omega_{e}\right)}\right] G_{e}(\omega+i \Gamma)\right\},
\end{aligned}
$$

and the third to

$$
\begin{aligned}
\Delta P_{\text {decay }}\left(\omega, \omega_{e}\right)= & E_{0}\left(\omega+\omega_{e}\right) \rho_{e} \Gamma G_{g}(\omega) q_{g} \\
& \times\left[\frac{q_{e}}{m} G_{e}\left(\omega+\omega_{e}\right)-\frac{q_{g}}{m} G_{g}\left(\omega+\omega_{e}\right)\right] \\
& \times\left[\frac{\gamma_{g}+\Gamma-2 i \omega}{\Gamma+i \omega_{e}} \frac{G_{e}(\omega+i \Gamma)}{G_{g}\left(\omega+\omega_{e}\right)}-1\right],
\end{aligned}
$$

where we assumed equal masses $m=m_{g}=m_{e}$. Since the decay term arises from the continuity condition on the dipolar oscillation at the moment of decay from the excited state back into the ground state, it vanishes in both limits $q_{g} \rightarrow 0$ and $\Gamma \rightarrow 0$. The conductivity $\Delta \sigma$ corresponding to the above polarization reads

$$
\begin{aligned}
& \Delta \sigma_{\text {vibrational }}\left(\omega, \omega_{e}\right)= \Delta \sigma_{\text {driven }}\left(\omega, \omega_{e}\right)+\Delta \sigma_{\text {excitation }}\left(\omega, \omega_{e}\right) \\
&+\Delta \sigma_{\text {decay }}\left(\omega, \omega_{e}\right), \\
& \Delta \sigma_{\text {driven }}\left(\omega, \omega_{e}\right)+\Delta \sigma_{\text {excitation }}\left(\omega, \omega_{e}\right) \\
&=-i \omega \frac{\rho_{e}}{\Gamma+i \omega_{e}}\left\{\frac{q_{g}}{m}\left(q_{e}-q_{g}\right) G_{g}\left(\omega+\omega_{e}\right)\right. \\
&\left.+\frac{q_{e}}{m}\left[q_{e}-q_{g} \frac{G_{g}\left(\omega+\omega_{e}\right)}{G_{e}\left(\omega+\omega_{e}\right)}\right] G_{e}(\omega+i \Gamma)\right\} \\
& \Delta \sigma_{\text {decay }}\left(\omega, \omega_{e}\right)=- i \omega \rho_{e} \Gamma G_{g}(\omega) q_{g} \\
& \times {\left[\frac{q_{e}}{m} G_{e}\left(\omega+\omega_{e}\right)-\frac{q_{g}}{m} G_{g}\left(\omega+\omega_{e}\right)\right] } \\
& \times {\left[\frac{\gamma_{g}+\Gamma-2 i \omega}{\Gamma+i \omega_{e}} \frac{G_{e}(\omega+i \Gamma)}{G_{g}\left(\omega+\omega_{e}\right)}-1\right] }
\end{aligned}
$$

\section{DRUDE ELECTRON GAS}

The above expressions apply to a driven, damped oscillator, which is temporarily excited into a state with different oscillator characteristics. That is to say, it does not apply to a Drude electron gas. However, our formalism is suitable to describe this case too. This is important for the interpretation of broadband pump-probe terahertz experiments, which probe interferences of the Drude electron gas and vibrational resonances. ${ }^{29}$ In this section we show what response may be expected from Drude electrons with finite lifetime $\tau_{c}=1 / \Gamma$. The electron cloud is created at time $t=t_{e}$ with (by definition) zero position and zero average velocity. After recombination with an ion to a neutral excited state, the Drude electron does not contribute to the photoinduced current anymore. Consequently, the position of a Drude electron with lifetime $\tau$ is "frozen" from time $t=t_{e}+\tau$ onwards,

$$
\begin{aligned}
x_{e}\left(t, t_{e}, \tau\right)= & \frac{q_{e}}{m_{e}}\left\{\theta\left(t_{e}+\tau-t\right) \theta\left(t-t_{e}\right)\right. \\
& \times \int_{t_{e}}^{t} d t^{\prime} E_{0}\left(t^{\prime}\right) G_{D}\left(t-t^{\prime}\right)+\theta\left(t-t_{e}-\tau\right) \\
& \left.\times \int_{t_{e}}^{t_{e^{+}+\tau}} d t^{\prime} E_{0}\left(t^{\prime}\right) G_{D}\left(t_{e}+\tau-t^{\prime}\right)\right\} .
\end{aligned}
$$

Here the Drude Green's function $G_{D}(t)$ is a solution of Eqs. 
(8) and (9) in the case of vanishing resonance frequency $\left(\omega_{\text {res }}=0\right)$,

$$
G_{D}(t)=\frac{\theta(t)}{\gamma_{D}}\left(1-e^{-\gamma_{D} t}\right),
$$

with $\gamma_{D}$ as the scattering time of the Drude electrons. Equation (21) cannot be Fourier transformed easily because the second term does not vanish for $t \rightarrow \infty$. However, its derivative with respect to the first time variable $t$,

$$
\dot{x}_{e}\left(t, t_{e}, \tau\right)=\frac{q_{e}}{m_{e}} \theta\left(t_{e}+\tau-t\right) \theta\left(t-t_{e}\right) \int_{t_{e}}^{t} d t^{\prime} E_{0}\left(t^{\prime}\right) \dot{G}_{D}\left(t-t^{\prime}\right),
$$

can be Fourier transformed without the problem of divergences. The resulting photoinduced current reads

$$
\begin{aligned}
& \Delta J_{\text {Drude }}\left(\omega, \omega_{e}\right)=E_{0}\left(\omega+\omega_{e}\right) \Delta \sigma_{\text {Drude }}\left(\omega, \omega_{e}\right), \\
& \Delta \sigma_{\text {Drude }}\left(\omega, \omega_{e}\right)=\frac{q_{e}^{2}}{m} \frac{\rho_{e}}{\left(\Gamma+i \omega_{e}\right)\left(\Gamma+\gamma_{D}-i \omega\right)},
\end{aligned}
$$

in agreement with Eq. (33) in Ref. 1. In comparing these expressions, please note that our couple $\left(\omega, \omega_{e}\right)$ corresponds to their $\left(-\omega, \omega_{p}\right)$, as a result of different definitions both in the delay times and in the Fourier transform.

\section{SPATIAL DEPENDENCE}

We now turn our attention to the spatial part of the problem. Using the Fourier convention of Eq. (17) we obtain for Eq. (1) the well-known expressions

$$
\begin{aligned}
& \frac{\partial H\left(z, \omega, t_{e}\right)}{\partial z}=i \omega\left\{\varepsilon_{0} E\left(z, \omega, t_{e}\right)+P\left(z, \omega, t_{e}\right)\right\}, \\
& \frac{\partial E\left(z, \omega, t_{e}\right)}{\partial z}=i \omega \mu_{0} H\left(z, \omega, t_{e}\right) .
\end{aligned}
$$

In the absence of excitation, these equations read

$$
\begin{aligned}
\frac{\partial H_{0}(z, \omega)}{\partial z} & =i \omega\left\{\varepsilon_{0} E_{0}(z, \omega)+P_{0}(z, \omega)\right\} \\
& =i \omega \varepsilon_{0} E_{0}(z, \omega)\left\{1+\chi_{g}(\omega)\right\}, \\
\frac{\partial E_{0}(z, \omega)}{\partial z} & =i \omega \mu_{0} H_{0}(z, \omega),
\end{aligned}
$$

with the polarization of Eq. (5) written as follows:

$$
\begin{aligned}
& P\left(z, \omega, t_{e}\right)=P_{0}(z, \omega)+\varepsilon_{0} \chi_{g}(\omega) \Delta E\left(z, \omega, t_{e}\right)+\Delta P\left(z, \omega, t_{e}\right), \\
& P_{0}(z, \omega)=\varepsilon_{0} \chi_{g}(\omega) E_{0}(z, \omega) .
\end{aligned}
$$

The susceptibility is linearly related to the ground-state Green's function,

$$
\varepsilon_{0} \chi_{g}(\omega)=\rho_{t} \frac{q_{g}^{2}}{m} G_{g}(\omega)
$$

in agreement with Eq. (6). Subtracting Eq. (26) from Eq. (25) yields the following equation for the difference fields:

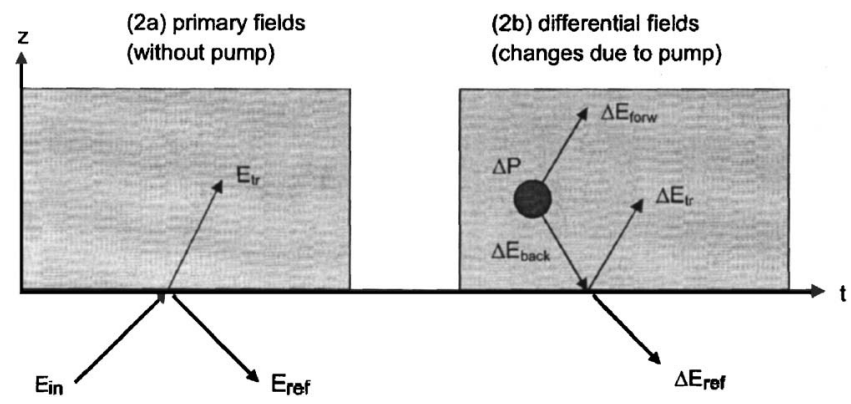

FIG. 2. Definition of the primary (a) and differential (b) fields. The vertical coordinate represents space $(z)$, the horizontal time $(t)$. The shaded area represents the sample, with the interface at $z=0$ marked with a thick line. The dark circle in the right diagram represents a (spatiotemporally) localized pump-induced polarization.

$$
\begin{aligned}
\frac{\partial \Delta H\left(z, \omega, t_{e}\right)}{\partial z}= & i \omega \varepsilon_{0} \Delta E\left(z, \omega, t_{e}\right)\left\{1+\chi_{g}(\omega)\right\} \\
& +i \omega \Delta P\left(z, \omega, t_{e}\right), \\
\frac{\partial \Delta E\left(z, \omega, t_{e}\right)}{\partial z}= & i \omega \mu_{0} \Delta H\left(z, \omega, t_{e}\right) .
\end{aligned}
$$

The separation of the total polarization into three terms as proposed in Eq. (27) has the important consequence that the differential fields $\Delta E$ and $\Delta H$ propagate with the same dielectric constant $\varepsilon_{0}\left\{1+\chi_{g}(\omega)\right\}$ as the primary fields $E_{0}$ and $H_{0}$, the only difference being the source term $\Delta P\left(z, \omega, t_{e}\right)$. The three polarization terms can be interpreted as follows: $P_{0}$ is the response of $N_{t}$ ground-state dipoles (i.e., as if none of them had been excited by the pump) to the primary field $E_{0}$; $\varepsilon_{0} \chi_{g}(\omega) \Delta E\left(z, \omega, t_{e}\right)$ is the response of $N_{t}$ ground-state dipoles to the differential field, and these two terms together describe the response of $N_{t}$ ground-state dipoles to the total field; the third term, the differential polarization $\Delta P$, describes the response of $N_{e}$ excited-state dipoles to the total field, minus the response of $N_{e}$ ground-state dipoles, thereby correcting for the fact that the first two terms assume $N_{t}$ ground-state dipoles instead of $N_{t}-N_{e}$.

From now on we replace the pump-free notation $E_{0}$ and $H_{0}$ by a different one which aims at describing the boundary conditions on the sample interface, defined as $z=0$. The incoming, reflected, and transmitted parts of the fields will be given by $E_{\mathrm{in}}, E_{\mathrm{ref}}$, and $E_{\mathrm{tr}}$, respectively, and likewise for $H$ [see Fig. 2(a)].

Moreover, the fields can be separated into a pump-free part describing the medium in the absence of a pump field, and a differential part $(\Delta E)$ describing the response to an exciting pump field. We write, for $z \leqslant 0$,

$$
\begin{aligned}
& E\left(z, \omega, t_{e}\right)=E_{\mathrm{in}}(z, \omega)+E_{\mathrm{ref}}(z, \omega)+\Delta E_{\mathrm{ref}}\left(z, \omega, t_{e}\right), \\
& H\left(z, \omega, t_{e}\right)=H_{\mathrm{in}}(z, \omega)+H_{\mathrm{ref}}(z, \omega)+\Delta H_{\mathrm{ref}}\left(z, \omega, t_{e}\right), \\
& P=0 .
\end{aligned}
$$

For $z \geqslant 0$, we write 


$$
\begin{aligned}
E\left(z, \omega, t_{e}\right)= & E_{\mathrm{tr}}(z, \omega)+\Delta E_{\mathrm{tr}}\left(z, \omega, t_{e}\right)+\Delta E_{\mathrm{back}}\left(z, \omega, t_{e}\right) \\
& +\Delta E_{\mathrm{forw}}\left(z, \omega, t_{e}\right), \\
H\left(z, \omega, t_{e}\right)= & H_{\mathrm{tr}}(z, \omega)+\Delta H_{\mathrm{tr}}\left(z, \omega, t_{e}\right)+\Delta H_{\mathrm{back}}\left(z, \omega, t_{e}\right) \\
& +\Delta H_{\mathrm{forw}}\left(z, \omega, t_{e}\right), \\
P\left(z, \omega, t_{e}\right)= & \varepsilon_{0} \chi_{g}(\omega) E\left(z, \omega, t_{e}\right)+\Delta P\left(z, \omega, t_{e}\right) .
\end{aligned}
$$

In general the polarization causes radiation in both the forward and backward directions. For the stationary case (without pump) the effects of the forward and backward radiated fields are fully taken into account by the index of refraction, resulting in a reflected field component at the air-sample interface. In the time-dependent case (with pump) the polarization produces a forward radiated electromagnetic field $\Delta E_{\text {forw }}$ and a backward radiated field $\Delta E_{\text {back}}$, respectively [see Fig. 2(b)]. On impinging onto the entrance interface of the sample, the backward traveling wave gives rise to a (backward) transmitted part, represented by the field $\Delta E_{\text {ref }} \mathrm{Eq}$. (30), and a (forward) reflected part, represented by the field $\Delta E_{\text {tr }}$ in Eq. (31).

Equations (25)-(31) lead to several derived expressions, which we subdivide into four cases: with and without pumping, and before and after the interface. One obtains:

(i) without pumping, before the interface $(z<0)$,

$$
\begin{aligned}
& \frac{\partial E_{\mathrm{in}}(z, \omega)}{\partial z}=i \omega \mu_{0} H_{\mathrm{in}}(z, \omega), \\
& \frac{\partial H_{\mathrm{in}}(z, \omega)}{\partial z}=i \omega \varepsilon_{0} E_{\mathrm{in}}(z, \omega), \\
& \frac{\partial E_{\mathrm{ref}}(z, \omega)}{\partial z}=i \omega \mu_{0} H_{\mathrm{ref}}(z, \omega), \\
& \frac{\partial H_{\mathrm{ref}}(z, \omega)}{\partial z}=i \omega \varepsilon_{0} E_{\mathrm{ref}}(z, \omega) ;
\end{aligned}
$$

(ii) without pumping, after the interface $(z>0)$,

$$
\begin{aligned}
& \frac{\partial E_{\mathrm{tr}}(z, \omega)}{\partial z}=i \omega \mu_{0} H_{\mathrm{tr}}(z, \omega), \\
& \frac{\partial H_{\mathrm{tr}}(z, \omega)}{\partial z}=i \omega \varepsilon_{0}\left\{1+\chi_{g}(\omega)\right\} E_{\mathrm{tr}}(z, \omega),
\end{aligned}
$$

where the boundary conditions for the air-sample interface imply

$$
\begin{aligned}
& E_{\text {in }}(0, \omega)+E_{\text {ref }}(0, \omega)=E_{\text {tr }}(0, \omega), \\
& H_{\text {in }}(0, \omega)+H_{\text {ref }}(0, \omega)=H_{\text {tr }}(0, \omega) ;
\end{aligned}
$$

(iii) with pumping, before the interface $(z<0)$,

$$
\begin{aligned}
& \frac{\partial \Delta E_{\mathrm{ref}}\left(z, \omega, t_{e}\right)}{\partial z}=i \omega \mu_{0} \Delta H_{\mathrm{ref}}\left(z, \omega, t_{e}\right), \\
& \frac{\partial \Delta H_{\mathrm{ref}}\left(z, \omega, t_{e}\right)}{\partial z}=i \omega \varepsilon_{0} \Delta E_{\mathrm{ref}}\left(z, \omega, t_{e}\right) ;
\end{aligned}
$$

(iv) with pumping, after the interface $(z>0)$,

$$
\begin{aligned}
& \frac{\partial}{\partial z} \Delta E_{\mathrm{tr}}\left(z, \omega, t_{e}\right)=i \omega \mu_{0} \Delta H_{\mathrm{tr}}\left(z, \omega, t_{e}\right) \\
& \frac{\partial}{\partial z} \Delta H_{\mathrm{tr}}\left(z, \omega, t_{e}\right)=i \omega \varepsilon_{0}\left\{1+\chi_{g}(\omega)\right\} \Delta E_{\mathrm{tr}}\left(z, \omega, t_{e}\right) \\
& \frac{\partial}{\partial z}\left\{\Delta E_{\mathrm{back}}\left(z, \omega, t_{e}\right)+\Delta E_{\mathrm{forw}}\left(z, \omega, t_{e}\right)\right\} \\
& \quad=i \omega \mu_{0}\left\{\Delta H_{\mathrm{back}}\left(z, \omega, t_{e}\right)+\Delta H_{\mathrm{forw}}\left(z, \omega, t_{e}\right)\right\} \\
& \quad \times\left\{\Delta E_{\mathrm{back}}\left(z, \omega, t_{e}\right)+\Delta E_{\mathrm{forw}}\left(z, \omega, t_{e}\right)\right\} \\
& \quad+i \omega \Delta P\left(z, \omega, t_{e}\right),
\end{aligned}
$$

with the corresponding boundary conditions,

$$
\begin{aligned}
& \Delta E_{\mathrm{ref}}\left(0, \omega, t_{e}\right)=\Delta E_{\mathrm{tr}}\left(0, \omega, t_{e}\right)+\Delta E_{\mathrm{back}}\left(0, \omega, t_{e}\right) \\
& \Delta H_{\mathrm{ref}}\left(0, \omega, t_{e}\right)=\Delta H_{\mathrm{tr}}\left(0, \omega, t_{e}\right)+\Delta H_{\mathrm{back}}\left(0, \omega, t_{e}\right) .
\end{aligned}
$$

Equations (32)-(34) (without pumping) are solved by the well-known expressions

$$
\begin{aligned}
& E_{\mathrm{in}}(z, \omega)=E_{\mathrm{in}}(0, \omega) e^{i \omega z / c}, \\
& H_{\mathrm{in}}(z, \omega)=c \varepsilon_{0} E_{\mathrm{in}}(0, \omega) e^{i \omega z / c}, \\
& E_{\mathrm{ref}}(z, \omega)=E_{\mathrm{ref}}(0, \omega) e^{-i \omega z / c}, \\
& H_{\mathrm{ref}}(z, \omega)=-c \varepsilon_{0} E_{\mathrm{ref}}(0, \omega) e^{-i \omega z / c}, \\
& E_{\mathrm{tr}}(z, \omega)=E_{\mathrm{tr}}(0, \omega) e^{i \omega n(\omega) z / c}, \\
& H_{\mathrm{tr}}(z, \omega)=c \varepsilon_{0} n(\omega) E_{\mathrm{tr}}(0, \omega) e^{i \omega n(\omega) z / c}, \\
& E_{\mathrm{tr}}(0, \omega)=\frac{2}{1+n(\omega)} E_{\mathrm{in}}(0, \omega), \\
& E_{\mathrm{ref}}(0, \omega)=\frac{1-n(\omega)}{1+n(\omega)} E_{\mathrm{in}}(0, \omega),
\end{aligned}
$$

with the index of refraction $n(\omega)$ defined by the relation $n^{2}(\omega)=1+\chi_{g}(\omega)$. Likewise, Eqs. (35)-(37) for the pumpinduced field changes are solved by 


$$
\begin{aligned}
& \Delta E_{\mathrm{ref}}\left(z, \omega, t_{e}\right)=\Delta E_{\mathrm{ref}}\left(0, \omega, t_{e}\right) e^{-i \omega z / c}, \\
& \Delta H_{\mathrm{ref}}\left(z, \omega, t_{e}\right)=-c \varepsilon_{0} \Delta E_{\mathrm{ref}}\left(0, \omega, t_{e}\right) e^{-i \omega z / c}, \\
& \Delta E_{\mathrm{tr}}\left(z, \omega, t_{e}\right)=\Delta E_{\mathrm{tr}}\left(0, \omega, t_{e}\right) e^{i \omega n(\omega) z / c}, \\
& \Delta H_{\mathrm{tr}}\left(z, \omega, t_{e}\right)=c \varepsilon_{0} n(\omega) \Delta E_{\mathrm{tr}}\left(0, \omega, t_{e}\right) e^{i \omega n(\omega) z / c}, \\
& \Delta E_{\mathrm{tr}}\left(0, \omega, t_{e}\right)=\frac{n(\omega)-1}{n(\omega)+1} \Delta E_{\mathrm{back}}\left(0, \omega, t_{e}\right), \\
& \Delta E_{\mathrm{ref}}\left(0, \omega, t_{e}\right)=\frac{2 n(\omega)}{n(\omega)+1} \Delta E_{\mathrm{back}}\left(0, \omega, t_{e}\right), \\
& \Delta E_{\text {back }}\left(z, \omega, t_{e}\right)+\Delta E_{\text {forw }}\left(z, \omega, t_{e}\right) \\
& =\int d z^{\prime} \Delta P\left(z^{\prime}, \omega, t_{e}\right) e\left(z-z^{\prime}, \omega\right), \\
& \Delta H_{\mathrm{back}}\left(z, \omega, t_{e}\right)+\Delta H_{\text {forw }}\left(z, \omega, t_{e}\right) \\
& =\int d z^{\prime} \Delta P\left(z^{\prime}, \omega, t_{e}\right) h\left(z-z^{\prime}, \omega\right) \text {. }
\end{aligned}
$$

The Green's functions $e(z, \omega)$ and $h(z, \omega)$ used to calculate the fields radiated by the polarized dipoles are defined by

$$
\begin{aligned}
& e(z, \omega)=\frac{i \omega \mu_{0} c}{2 n(\omega)} e^{i \omega n(\omega)|z| / c}, \\
& h(z, \omega)=\frac{1}{2} \omega \frac{z}{|z|} e^{i \omega n(\omega)|z| / c},
\end{aligned}
$$

providing the solution to the equations

$$
\begin{aligned}
& \frac{\partial e(z, \omega)}{\partial z}=i \omega \mu_{0} h(z, \omega), \\
& \frac{\partial h(z, \omega)}{\partial z}=i \omega\left\{\varepsilon_{0} n^{2}(\omega) e(z, \omega)+\delta(z)\right\} .
\end{aligned}
$$

We now consider the spatiotemporal dependence of the density of excited states, $\rho_{e}(z, t)$. In the case of a finite duration of the pump pulse the excited-state population is determined by the relation

$$
\frac{\partial}{\partial t} \rho_{e}(z, t)=S(z, t)-\Gamma \rho_{e}(z, t)
$$

The rate of charge carrier production, $S(z, t)$, is given by

$$
S(z, t)=\rho_{e} \theta(z) e^{-\alpha z} I\left(t-n_{p} z / c\right),
$$

with $\alpha$ the reciprocal penetration depth of the pump beam, and $I\left(t-n_{p} z / c\right)$ the intensity profile of the pump beam propagating through the sample assume without dispersion and a corresponding group velocity $c / n_{p}$. The "effective index of refraction for group propagation" $n_{p}$ is related to the index of refraction $n(\omega)$ as

$$
n_{p}=n\left(\omega_{\text {pump }}\right)-\left.\omega_{\text {pump }} \frac{d n(\omega)}{d \omega}\right|_{\omega_{\text {pump }}},
$$

with $\omega_{\text {pump }}$ as the center radial frequency of the pump beam. In frequency domain the relative density of excited dipoles can be written as follows:

$$
\rho_{e}(z, \omega)=\rho_{e}(\omega) I(\omega) \theta(z) \exp \left(-\alpha z+i \omega n_{p} z / c\right),
$$

with $\rho_{e}(\omega)=\rho_{e} /(\Gamma-i \omega)$. At this point one has all ingredients to perform the spatial integration over the Green's functions in Eq. (39). When the sample is thick enough to absorb all the excitation light we do not have to take into account the excitation profile generated by (backward traveling) pump light reflected off the exit interface of the sample. In the thick-sample approximation the integration limits may be extended to infinity,

$$
\begin{aligned}
& \Delta E_{\text {forw }}(z \rightarrow \infty, \omega, q)=\int_{0}^{\infty} d z^{\prime} \Delta P\left(z^{\prime}, \omega, q\right) e\left(z-z^{\prime}, \omega\right), \\
& \Delta E_{\text {back }}(z=0, \omega, q)=\int_{0}^{\infty} d z^{\prime} \Delta P\left(z^{\prime}, \omega, q\right) e\left(-z^{\prime}, \omega\right) .
\end{aligned}
$$

Using Eq. (18) for $\Delta P_{\text {driven }}$, Eq. (45) for $\rho_{e}$, Eq. (38) for $E_{\mathrm{tr}}$, Eq. (39) for $\Delta E_{\mathrm{back}}$, and $\Delta E_{\mathrm{tr}}$, and Eq. (40) for $e$, we obtain the following solution for the transmitted Terahertz wave $\Delta E \equiv \Delta E_{\text {forw }}+\Delta E_{\mathrm{tr}}$ :

$$
\begin{aligned}
& \frac{\Delta E\left(z, \omega, \omega_{e}\right)}{E_{\mathrm{tr}}\left(z=0, \omega+\omega_{e}\right)}= \frac{\Delta \sigma\left(\omega, \omega_{e}\right)}{2 n(\omega)} I\left(-\omega_{e}\right) e^{i \omega n(\omega) z / c} \\
& \times\left\{\frac{1}{F^{(+)}}+\frac{n(\omega)-1}{n(\omega)+1} \frac{1}{F^{(-)}}\right\}, \\
& F^{( \pm)} \equiv \alpha c-i \omega_{e} n_{p}-i\left(\omega+\omega_{e}\right) n\left(\omega+\omega_{e}\right) \pm i \omega n(\omega),
\end{aligned}
$$

with the conductivity $\Delta \sigma$ defined above in Eq. (20). For an infinitely long sampling window $\left(T_{w} \rightarrow \infty\right)$ the measured fields are related to the real fields as [see Eq. (25)]

$$
\frac{\Delta E^{\text {meas }}\left(z, \omega, \omega_{e}\right)}{E_{\mathrm{tr}}^{\text {meas }}\left(z=0, \omega+\omega_{e}\right)}=\frac{\psi(\omega)}{\psi\left(\omega+\omega_{e}\right)} \frac{\Delta E\left(z, \omega, \omega_{e}\right)}{E_{\mathrm{tr}}\left(z=0, \omega+\omega_{e}\right)} .
$$

This introduces the detector response into Eq. (47) when written in terms of the measured fields. Different expressions for the spatial factor $F^{( \pm)}$in Eq. (47), applying to thin samples, or to samples with low absorption, can be found in the seminal papers. ${ }^{23}$

\section{NUMERICAL VALIDATION}

In spite of the simplicity of our model (a driven damped harmonic oscillator) the resulting expressions for the polarization in the frequency domain, Eqs. (18) and (19), are quite cumbersome, and we felt the need to validate them independently by comparing them with straightforward numerical solutions for a specific set of conditions. These conditions are (i) the driving field is a continuous-wave field of the form $E_{0}(t)=E_{0} \cos \omega_{0} t$; (ii) the effective charges in the excited and ground states are equal $\left(q_{e}=q_{g}\right)$ such that the polarization [Eqs. (18) and (19)] $\Delta P\left(t, t_{e}\right)$ is linearly related to the posi- 


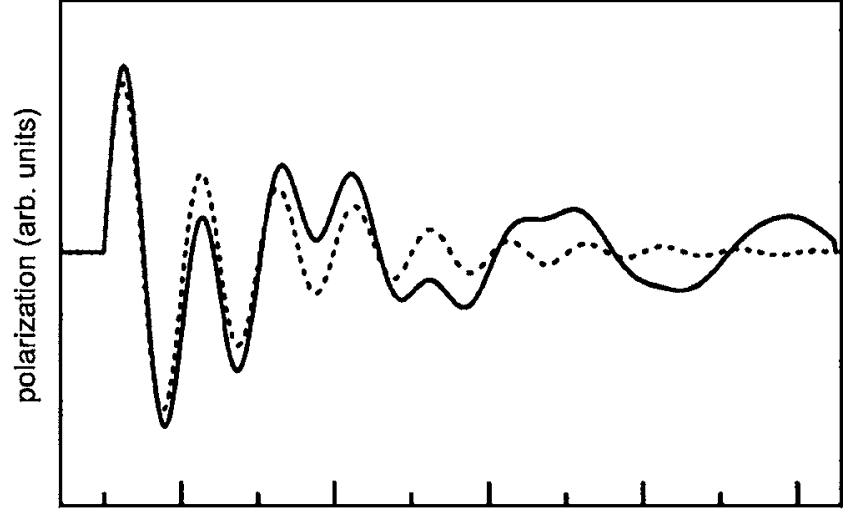

time (units of driving field period)

FIG. 3. Comparison of two analytical solutions with a single numerical solution of Eqs. (12)-(15). The vertical axis displays the time-shifted polarization $\Delta P\left(t-t_{e}, t_{e}\right)$. The time unit on the horizontal axis is the period of the driving field $2 \pi / \omega_{0}$. (i) The full analytical expression [Eqs. (18) and (19)] is indistinguishable from the numerical calculation (continuous line for both of them). (ii) The analytical solution excluding the decay term [Eq. (19)] is represented by the dotted line. At short times after excitation it follows the exact solution closely. After four cycles of the driving field (roughly the lifetime of the excited states) the oscillatory frequency is unchanged, where the exact solution shows a gradual transition from the driving frequency of the field to the resonance frequency of the ground state (the resonance frequency was set at one-third the frequency of the driving field).

tion difference of the excited- and ground-state oscillators $x_{e}-x_{g}$; (iii) the resonance frequency of the ground state is one-third the frequency of the driving field; and (iv) the damping rates are equal in the excited and ground states.

The analytical solution for the position in the twodimensional (2D) time domain was retrieved from the polarization by a one-dimensional numerical Fourier transform from $\omega$ to $t$; the transform from $\omega_{e}$ to $t_{e}$ was done trivially using the delta function of the driving field, $E_{0}\left(\omega+\omega_{e}\right)$ $=\pi E_{0}\left[\delta\left(\omega+\omega_{e}+\omega_{0}\right)+\delta\left(\omega+\omega_{e}-\omega_{0}\right)\right]$. The numerical calculations are based on Eqs. (12)-(15), with the Green's function defined in the Appendix [Eq. (A4)]. The results are plotted in Fig. 3.

The numerical results are indistinguishable from the analytical ones. However, when the decay term $\Delta P_{\text {decay }}$ is neglected, discrepancies arise. This proves, along with the derivation presented in Sec. V, that our solutions can be trusted.

\section{DISCUSSION}

The terahertz conductivity for a Drude electron gas, Eq. (24), reproduces an expression already published in the literature. ${ }^{1}$ This is not the case for Eq. (20) for the vibrational conductivity, which differs slightly from its corresponding expression in the literature [Eq. (50) in Sec. IIIC in Ref. 1]: where the literature has a factor of $\Gamma-i \omega$ (our notation), whereas we have a factor of $-i \omega$. The difference between these two expressions becomes apparent upon considering the response of the oscillators to a dc electric field.

The response of the current $\Delta J_{\mathrm{dc}}\left(\omega, \omega_{e}\right)$ to the dc field $E_{\mathrm{dc}}(\omega)=2 \pi E_{0} \delta(\omega)$ reads

$$
\begin{aligned}
\Delta J_{\mathrm{dc}}\left(\omega, \omega_{e}\right) & =\Delta \sigma\left(\omega, \omega_{e}\right) E_{\mathrm{dc}}\left(\omega+\omega_{e}\right) \\
& =2 \pi \delta\left(\omega+\omega_{e}\right) \Delta \sigma\left(\omega, \omega_{e}\right) E_{0} .
\end{aligned}
$$

Since a dc field has no phase, in the time domain this current depends on its temporal variables only through the difference $t-t_{e}$. This is adequately reflected by the introduction of wiggled, single-variable functions as follows:

$$
\begin{aligned}
& \Delta \widetilde{J}_{\mathrm{dc}}(t) \equiv \Delta J_{\mathrm{dc}}(t, 0), \\
& \Delta \widetilde{J}_{\mathrm{dc}}\left(t-t_{e}\right)=\Delta J_{\mathrm{dc}}\left(t, t_{e}\right)=\frac{1}{2 \pi} \int d \omega e^{-i \omega\left(t-t_{e}\right)} \Delta \widetilde{J}_{\mathrm{dc}}(\omega),
\end{aligned}
$$

$$
\Delta \widetilde{J}_{\mathrm{dc}}(\omega)=E_{0} \Delta \sigma(\omega,-\omega) .
$$

The integrated current surge follows as

$$
\int_{-\infty}^{\infty} d t \Delta \widetilde{J}_{\mathrm{dc}}\left(t-t_{e}\right)=\Delta \widetilde{J}_{\mathrm{dc}}(\omega=0)=E_{0} \Delta \sigma(0,0) .
$$

On the other hand it can be written in terms of the polarization as

$$
\begin{aligned}
& \begin{aligned}
\int_{-\infty}^{\infty} d t \Delta \widetilde{J}_{\mathrm{dc}}\left(t-t_{e}\right) & =\int_{-\infty}^{\infty} d t\left\{\frac{d}{d t} \Delta \widetilde{P}_{\mathrm{dc}}\left(t-t_{e}\right)\right\} \\
& =\Delta \widetilde{P}_{\mathrm{dc}}(\infty)-\Delta \widetilde{P}_{\mathrm{dc}}(-\infty)
\end{aligned} \\
& \Delta \widetilde{P}_{\mathrm{dc}}(t) \equiv \Delta P_{\mathrm{dc}}(t, 0) .
\end{aligned}
$$

Since the vibrational motion of the oscillator is identical to its motion in the ground state at the times $t \rightarrow \pm \infty$, it is clear that the above polarization difference must vanish. Hence we find that, for the vibrational model defined in Sec. V, the 2D-frequency dependent conductivity $\Delta \sigma\left(\omega, \omega_{e}\right)$ must obey the relation

$$
\Delta \sigma_{\text {vibrational }}(0,0)=0 .
$$

This is indeed the case for our vibrational expression, Eq. (20). Relation (53) does not apply to Eq. (50) in Sec. IIIC in Ref. 1. From this we deduce that their model does not describe a vibrational oscillator excited at time $t=t_{e}$ and decayed back to the ground state at a later time $t=t_{e}+\tau$ with some distribution of finite lifetimes $\tau$.

Relation (53) does not apply to the Drude conductivity [Eq. (24)] because from Eq. (21) it can be seen that the quantities $x_{e}\left(t \rightarrow \infty, t_{e}, \tau\right)$ and $\Delta \widetilde{P}_{\mathrm{dc} \text {,Drude }}(t \rightarrow \infty)$ do not vanish.

Finally, we added a decay term to the vibrational conductivity published in the literature. ${ }^{1}$ The decay term corresponds to the vibrational motion of an oscillator after the decay of the excited state to the ground state. A numerical calculation confirms our analytical results.

\section{ACKNOWLEDGMENTS}

This work is part of the research program of the "Stichting voor Fundamenteel Onderzoek der Materie (FOM)," which is financially supported by the "Nederlandse Organi- 
satie voor Wetenschappelijk Onderzoek (NWO)." We are grateful for helpful discussions with Petr Kuzel.

\section{APPENDIX: CONTINUITY CONDITIONS}

Equation (12) describes the motion of a damped, driven oscillator $x_{e}\left(t, t_{e}, \tau\right)$ during three separate periods. Before the moment of excitation, for $t<t_{e}$, the motion of the oscillator is determined by the ground-state dynamics [Eq. (8)]. After excitation, for $t>t_{e}$, the oscillator obeys excited-state dynamics [Eq. (10)], until the moment that the excitation spontaneously decays back into the ground state, at $t=t_{e}+\tau$. From that moment onwards, the oscillator obeys ground-state dynamics again.

For $t<t_{e}$, the solution reads

$$
x_{e}\left(t, t_{e}, \tau\right)=\theta\left(t_{e}-t\right) \frac{q_{s}}{m_{g}} \int_{-\infty}^{t} d t^{\prime} E_{0}\left(t^{\prime}\right) G_{g}\left(t-t^{\prime}\right),
$$

and for $t_{e}<t<t_{e}+\tau$,

$$
\begin{aligned}
x_{e}\left(t, t_{e}, \tau\right)= & \theta\left(t_{e}+\tau-t\right) \theta\left(t-t_{e}\right) \\
& \times\left\{\frac{q_{e}}{m_{e}} \int_{-\infty}^{t} d t^{\prime} E_{0}\left(t^{\prime}\right) G_{e}\left(t-t^{\prime}\right)-e\left(t, t_{e}\right)\right\},
\end{aligned}
$$

with

$$
e\left(t, t_{e}\right)=e_{1}\left(t_{e}\right) G_{e}\left(t-t_{e}\right)+e_{2}\left(t_{e}\right) \dot{G}_{e}\left(t-t_{e}\right)
$$

as the homogeneous solution of the excited-state dynamics. Using $\Omega_{e}=\sqrt{\omega_{\text {res }, e}^{2}-\gamma_{e}^{2} / 4}$, the Green's function for the underdamped case $\left(\omega_{\text {res }, e}>\gamma_{e} / 2\right)$ can be written as

$$
G_{e}(t)=\theta(t) \frac{\sin \Omega_{e} t}{\Omega_{e}} e^{-\gamma_{e} t / 2},
$$

with the limits

$$
\begin{aligned}
& \lim _{t \downarrow 0} G_{e}(t)=0, \\
& \lim _{t \downarrow 0} \dot{G}_{e}(t)=1, \\
& \lim _{t \downarrow 0} \ddot{G}_{e}(t)=-\gamma_{e},
\end{aligned}
$$

where the dot indicates a derivative to the (first) time variable. In the case of overdamped dynamics the sine and cosine functions in Eq. (A4) must be replaced with their hyperbolic siblings, and $\Omega_{e \text {,overdamped }}=\sqrt{\gamma_{e}^{2} / 4-\omega_{\text {res }, e}^{2}}$. Continuity of both position and velocity at $t=t_{e}$,

$$
\begin{aligned}
& \lim _{t \uparrow t_{e}} x_{e}\left(t, t_{e}, \tau\right)=\lim _{t \downarrow t_{e}} x_{e}\left(t, t_{e}, \tau\right), \\
& \lim _{t \uparrow t_{e}} \dot{x}_{e}\left(t, t_{e}, \tau\right)=\lim _{t \downarrow t_{e}} \dot{x}_{e}\left(t, t_{e}, \tau\right),
\end{aligned}
$$

when applied to the solutions (A1) and (A2), results in two conditions for the homogeneous amplitudes $e_{1,2}$,

$$
\begin{aligned}
& \frac{q_{g}}{m_{g}} \int d t^{\prime} E_{0}\left(t^{\prime}\right) G_{g}\left(t_{e}-t^{\prime}\right) \\
& \quad=\frac{q_{e}}{m_{e}} \int d t^{\prime} E_{0}\left(t^{\prime}\right) G_{e}\left(t_{e}-t^{\prime}\right)-e\left(t_{e}, t_{e}\right), \\
& \left.\frac{q_{g}}{m_{g}} \int d t^{\prime} E_{0}\left(t^{\prime}\right) \dot{G}_{g}\left(t-t^{\prime}\right)\right|_{t=t_{e}}||_{t=t_{e}}-\left.\dot{e}\left(t, t_{e}\right)\right|_{t=t_{e}} .
\end{aligned}
$$

Consequently, the amplitudes must have the values

$$
\begin{aligned}
& e_{1}\left(t_{e}\right)=\int d t^{\prime} E_{0}\left(t^{\prime}\right)\left\{\Delta \dot{K}\left(t_{e}-t^{\prime}\right)+\gamma_{e} \Delta K\left(t_{e}-t^{\prime}\right)\right\}, \\
& e_{2}\left(t_{e}\right)=\int d t^{\prime} E_{0}\left(t^{\prime}\right) \Delta K\left(t_{e}-t^{\prime}\right),
\end{aligned}
$$

with

$$
\Delta K(t) \equiv \frac{q_{e}}{m_{e}} G_{e}(t)-\frac{q_{g}}{m_{g}} G_{g}(t) .
$$

In the same way we can derive the coefficients for the solution at times after decay of the excited state $t>t_{e}+\tau$,

$$
\begin{aligned}
x_{e}\left(t, t_{e}, \tau\right)= & \theta\left(t-t_{e}-\tau\right) \\
& \times\left\{\frac{q_{g}}{m_{g}} \int d t^{\prime} E_{0}\left(t^{\prime}\right) G_{g}\left(t-t^{\prime}\right)+g\left(t, t_{e}, \tau\right)\right\},
\end{aligned}
$$

with

$$
g\left(t, t_{e}, \tau\right)=g_{1}\left(t_{e}, \tau\right) G_{g}\left(t-t_{e}-\tau\right)+g_{2}\left(t_{e}, \tau\right) \dot{G}_{g}\left(t-t_{e}-\tau\right)
$$

Continuity at time $t=t_{e}+\tau$ yields

$$
\begin{aligned}
& \frac{\underline{q_{e}}}{m_{e}} \int d t^{\prime} E_{0}\left(t^{\prime}\right) G_{e}\left(t_{e}+\tau-t^{\prime}\right)-e\left(t_{e}+\tau, t_{e}\right) \\
& \quad=\frac{q_{g}}{m_{g}} \int d t^{\prime} E_{0}\left(t^{\prime}\right) G_{g}\left(t_{e}+\tau-t^{\prime}\right)+g\left(t_{e}+\tau, t_{e}, \tau\right), \\
& \left.\frac{q_{e}}{m_{e}} \int d t^{\prime} E_{0}\left(t^{\prime}\right) \dot{G}_{e}\left(t-t^{\prime}\right)\right|_{t=e_{e}+\tau}-\left.\dot{e}\left(t, t_{e}\right)\right|_{t=t_{e}+\tau} \\
& \quad=\left.\frac{q_{g}}{m_{g}} \int d t^{\prime} E_{0}\left(t^{\prime}\right) \dot{G}_{g}\left(t-t^{\prime}\right)\right|_{t=t_{e}+\tau}+\left.\dot{g}\left(t, t_{e}, \tau\right)\right|_{t=t_{e}+\tau},
\end{aligned}
$$

with the corresponding solutions for the homogeneous amplitudes $g_{1,2}\left(t_{e}, \tau\right)$ : 


$$
\begin{aligned}
g_{1}\left(t_{e}, \tau\right)= & \int d t^{\prime} E_{0}\left(t^{\prime}\right)\left\{\Delta \dot{K}\left(t_{e}+\tau-t^{\prime}\right)+\gamma_{g} \Delta K\left(t_{e}+\tau-t^{\prime}\right)\right\} \\
& -e_{1}\left(t_{e}\right)\left\{\dot{G}_{e}(\tau)+\gamma_{g} G_{e}(\tau)\right\}-e_{2}\left(t_{e}\right)\left\{\ddot{G}_{e}(\tau)+\gamma_{g} \dot{G}_{e}(\tau)\right\},
\end{aligned}
$$

$$
\begin{aligned}
g_{2}\left(t_{e}, \tau\right)= & \int d t^{\prime} E_{0}\left(t^{\prime}\right) \Delta K\left(t_{e}+\tau-t^{\prime}\right) \\
& -e_{1}\left(t_{e}\right) \dot{G}_{e}(\tau)-e_{2}\left(t_{e}\right) \ddot{G}_{e}(\tau) .
\end{aligned}
$$

Given the coefficients $e_{1}\left(t_{e}\right), e_{2}\left(t_{e}\right), g_{1}\left(t_{e}, \tau\right)$, and $g_{2}\left(t_{e}, \tau\right)$ of the homogeneous solutions [Eqs. (A3) and (A10)], the position of the excited oscillator [Eq. (12)] is determined at all times $t, t_{e}$, and $\tau$. Threefold integration over the lifetime $\tau$ [Eq. (15)], and time delays $t$ and $t_{e}$ [Eq. (17)] yields the polarization in $2 \mathrm{D}$-frequency domain.

The homogeneous amplitudes $e_{1}\left(t_{e}\right), e_{2}\left(t_{e}\right), g_{1}\left(t_{e}, \tau\right)$, and $g_{2}\left(t_{e}, \tau\right)$ need only slight adaptation when a difference in equilibrium position $\Delta x$ of the excited and ground-state oscillators is taken into account. One needs the following additions to the coefficients:

$$
\begin{aligned}
& \Delta e_{1}\left(t_{e}\right)=\gamma_{e} \Delta x, \\
& \Delta e_{2}\left(t_{e}\right)=\Delta x, \\
& \Delta g_{1}\left(t_{e}, \tau\right)=\gamma_{g} \Delta x, \\
& \Delta g_{2}\left(t_{e}, \tau\right)=\Delta x .
\end{aligned}
$$

This results in a fourth term in the total polarization [cf. Eqs. (16), (18), and (19)],

$$
\begin{aligned}
\Delta P_{\text {equilibrium }}\left(\omega, \omega_{e}\right) \\
=2 \pi \Delta x \delta\left(\omega+\omega_{e}\right) \rho_{e} E_{0}\left(\omega+\omega_{e}\right) \\
\quad \times\left\{q _ { g } \Gamma G _ { g } ( \omega ) \left\{\frac{\gamma_{g}-i \omega}{\Gamma-i \omega}-\left(\gamma_{e}+\Gamma-i \omega\right)\right.\right. \\
\left.\quad \times\left(\gamma_{g}+\Gamma-2 i \omega\right) G_{e}(\omega+i \Gamma)\right\}-\frac{q_{e}}{\Gamma+i \omega_{e}} \\
\left.\quad \times\left[1-\frac{G_{e}(\omega+i \Gamma)}{G_{e}\left(\omega+\omega_{e}\right)}\right]\right\} .
\end{aligned}
$$

These terms can be readily recognized from the 2Dfrequency conductivity due to the delta function (broadened in practice by the time resolution of the experimental setup), yielding diagonal lines at $\omega=-\omega_{e}$.
${ }^{1}$ H. Nemec, F. Kadlec, S. Surendran, P. Kuzel, and P. Jungwirth, J. Chem. Phys. 122, 104503 (2005).

${ }^{2}$ R. H. M. Groeneveld and D. Grischkowsky, J. Opt. Soc. Am. B 11, 2502 (1994).

${ }^{3}$ G. Haran, W.-D. Sun, K. Wynne, and R. M. Hochstrasser, Chem. Phys. Lett. 274, 365 (1997).

${ }^{4}$ M. Schall and P. U. Jepsen, Opt. Lett. 25, 13 (2000).

${ }^{5}$ J. Zielbauer and M. Wegener, Appl. Phys. Lett. 9, 1223 (1996).

${ }^{6}$ E. Knoesel, M. Bonn, J. Shan, F. Wang, and T. F. Heinz, J. Chem. Phys. 121, 394 (2004).

${ }^{7}$ O. Ostroverkhova, D. G. Cooke, F. A. Hegmann, J. E. Anthony, V. Podzorov, M. E. Gershenson, O. D. Jurchescu, and T. T. M. Palstra, Appl. Phys. Lett. 88, 162101 (2006).

${ }^{8}$ K. Reimann, R. P. Smith, A. M. Weiner, T. Elsaesser, and M. Woerner, Opt. Lett. 28, 471 (2003).

${ }^{9}$ V. K. Thorsmolle, R. D. Averitt, X. Chi, D. J. Hilton, D. L. Smith, A. P. Ramirez, and A. J. Taylor, Appl. Phys. Lett. 84, 891 (2004).

${ }^{10}$ G. M. Turner, C. A. Schmuttenmaer, and M. C. Beard, J. Phys. Chem. B 106, 7146 (2002)

${ }^{11}$ J. Shan, A. Nahata, and T. F. Heinz, J. Nonlinear Opt. Phys. Mater. 11, 31 (2002).

${ }^{12}$ E. Hendry, J. M. Schins, L. P. Candeias, L. D. A. Siebbeles, and M. Bonn, Phys. Rev. Lett. 92, 196601 (2004).

${ }^{13}$ M. C. Asplund, M. T. Zanni, and R. M. Hochstrasser, Proc. Natl. Acad. Sci. U.S.A. 97, 8219 (2000).

${ }^{14}$ S. Ogawa, H. Nagano, H. Petek, and A. P. Heberle, Phys. Rev. Lett. 78, 1339 (1997).

${ }^{15}$ J. Shan, F. Wang, E. Knoesel, M. Bonn, and T. F. Heinz, Phys. Rev. Lett. 90, 247401 (2003).

${ }^{16}$ E. Hendry, F. Wang, J. Shan, T. F. Heinz, and M. Bonn, Phys. Rev. B 69, 081101 (2004).

${ }^{17}$ R. Huber, C. Kübler, S. Tübel, A. Leitenstorfer, Q. T. Vu, H. Haug, F. Köhler, and M.-C. Amann, Phys. Rev. Lett. 94, 027401 (2005).

${ }^{18}$ L. Perfetti, T. Kampfrath, F. Schapper, A. Hagen, T. Hertel, C. M. Aguirre, P. Desjardins, R. Martel, C. Frischkorn, and M. Wolf, Phys. Rev. Lett. 96, 027401 (2006).

${ }^{19}$ M. C. Beard and C. A. Schmuttenmaer, J. Chem. Phys. 114, 2903 (2001).

${ }^{20}$ M. C. Beard, G. M. Turner, and C. A. Schmuttenmaer, Phys. Rev. B 62, 15764 (2000)

${ }^{21}$ M. C. Beard, G. M. Turner, and C. A. Schmuttenmaer, J. Appl. Phys. 90, 5915 (2001)

${ }^{22}$ K. P. H. Lui and F. A. Hegmann, Appl. Phys. Lett. 78, 3478 (2001).

${ }^{23}$ H. Nemec, F. Kadlec, and P. Kuzel, J. Chem. Phys. 117, 8454 (2002).

${ }^{24}$ H. Nemec, F. Kadlec, C. Kadlec, P. Kuzel, and P. Jungwirth, J. Chem. Phys. 122, 104504 (2005).

${ }^{25}$ A. Nahata, A. S. Weling, and T. F. Heinz, Appl. Phys. Lett. 69, 2321 (1996).

${ }^{26}$ B. Salem, D. Morris, V. Aimez, J. Beauvais, and D. Houde, Semicond. Sci. Technol. 21, 283 (2006).

${ }^{27}$ J. T. Kindt and C. A. Schmuttenmaer, J. Chem. Phys. 110, 8589 (1999).

${ }^{28}$ E. Hendry, M. Koeberg, J. M. Schins, H. K. Nienhuys, V. Sundstrom, L. D. A. Siebbeles, and A. Bonn, Phys. Rev. B 71, 125201 (2005).

${ }^{29}$ M. Koeberg, E. Hendry, J. M. Schins, H. A. v. Laarhoven, C. F. J. Flipse, K. Reimann, M. Woerner, T. Elsaesser, and M. Bonn, Phys. Rev. B 75, 195216 (2007). 